\title{
PERCEPÇÃO DO USO DE URINA ENTRE AGRICULTORES FAMILIARES DE SERRINHA-BA
}

\section{PERCEPTION OF THE USE OF URINE AMONG THE FAMILY FARMERS IN SERRINHA-BA}

\section{Mathurin François}

Mestre em Engenharia Civil e Ambiental, Universidade Estadual de Feira de Santana. (mathurin.francois@yahoo.fr)

Eduardo Henrique Borges Cohim Silva.

Doutor em Energia e Meio Ambiente, Universidade Estadual de Feira de Santana. (edcohim@gmail.com)

\section{Sílvio Roberto Magalhães Orrico.}

Doutor em Saúde Pública, Universidade Estadual de Feira de Santana. (srm.orrico@gmail.com)

Maria Auxiliadora Freitas dos Santos.

Doutorando em Meio Ambiente, Instituto Federal Baiano. (dorafreitas2004@yahoo.com.br)

Submissão: 24 de Agosto de 2019Aceite: 18 de Novembro de 2019

\section{Resumo}

O uso de urina humana na agricultura é uma alternativa promissora para reduzir a poluição ambiental e a importação de fertilizantes industriais, melhorar a qualidade do solo e aumentar a produção agrícola, porém, sua aceitação varia em função do tipo de culturas. Este trabalho avaliou a percepção de agricultores familiares do Semiárido a respeito do uso de urina humana na agricultura. Foram entrevistados 210 agricultores, escolhidos aleatoriamente, por meio da aplicação de um questionário, e também apresentadas diferentes amostras com urina pura, urina diluída e compostos de urina. Houve aceitação inicial de urina como fertilizante por $67,7 \%$ dos entrevistados que optaram pelo uso em culturas ingeridas cozidas ou sem contato direto com a urina. Como impedimento ao uso, os entrevistados indicaram: risco à saúde $(54,3 \%)$, nojo $(27,1 \%)$ e mau cheiro $(24,8 \%)$. A aceitação inicial de se utilizar a urina caiu para 31,5\% quando da apresentação de amostras de urina pura ou diluída, mas alcançou um valor de aceitação de 53,8\% para a amostra misturada com cinza, indicando que sua descaracterização pode ser uma estratégia para torná-la mais aceitável.

Palavras chaves: reúso de urina humana; percepção dos agricultores; saneamento ecológico.

\section{Abstract}

The use of human urine in agriculture is a promising alternative to reduce environmental pollution and importation of industrial fertilizers as well as to improve agricultural soil quality and the crop yields. However, its acceptance varies from cultures to cultures. This work evaluated the perception of family farmers of Semiarid regarding the use of human urine in agriculture. 210 farmers were selected randomly to be interviewed through the application of questionnaire. The urine was accepted by $67.7 \%$ to fertilize plants that are cooked ingested or without direct contact with it. The health risk (54.3\%), disgust $(27.1 \%)$ and bad smell $(24.8 \%)$ were the main barriers. Previous acceptance dropped to $31.5 \%$ after presentation of pure and diluted urine samples, but reached $53.8 \%$ when the urine was mixed with ashes. This result showed that the decharacterization of urine should be a good strategy to make it more acceptable.

Key words: Reuse of human urine; farmers' perception; ecological sanitation.

\section{INTRODUÇÃO}

Devido à forte urbanização e ao crescimento populacional, muitos problemas ambientais têm ocorrido, tanto nas áreas urbanas como nas áreas rurais, onde os esgotos domésticos ou urina e fezes são encaminhados para corpos d'água sem tratamento prévio. $O$ lançamento direto ou indireto desses resíduos 
causa contaminação e eutrofização, a presença de patógenos e de nutrientes, respectivamente, - que causa prejuízos econômicos devido à perda da qualidade da água, uma vez que eleva os custos de tratamento da água e reduz as espécies de peixes de melhor valor comercial. Além disso, causa doenças de veiculação hídrica, o que resulta em gastos para o serviço de saúde.

Dados da carga global de doenças, divulgados em IHME (Institute for Health Metrics and Evaluation) para o ano de 2016, apontam para um percentual de $3,4 \%$ (80 milhões de DALY ${ }^{1}$ ) atribuíveis à falta de atendimento com sistemas adequados de abastecimento de água e de esgotamento sanitário (WEIDEMA; FANTKE, 2018). Os mesmos autores relatam que o fator de risco subnutrição é responsável por 4,6\% (109 milhões de DALY).

O atual modelo convencional de saneamento, de fato, reduz a poluição hídrica e a transmissão de doenças, porém, sua concepção é baseada em fluxo linear da água, nutriente e energia, considerado atualmente como um modelo que tende a exaurir a si e os recursos naturais. Essa situação torna-se mais crítica em regiões semiáridas, onde há pouca disponibilidade de água e uma grande quantidade de pequenas propriedades, tendo como principal atividade econômica a agricultura familiar, cuja produtividade ainda é baixa, o que implica em um necessário uso de fertilizantes químicos e, muitas vezes, em gastos de importação desses produtos e também dos próprios alimentos.

O enfrentamento dessas questões se liga diretamente a dois dos 17 objetivos do desenvolvimento sustentável definidos pelo Banco Mundial (WORLD BANK, 2018): o objetivo 2, que se refere à erradicação da fome, e o objetivo 6, que defende a universalização do abastecimento de água e esgotamento sanitário.

No que tange à produção de alimentos, observa-se, em muitas partes do mundo, uma grande necessidade de importação de

${ }^{1}$ Anos de vida com ajustamento pela deficiência fertilizantes químicos, fazendo com que a alimentação dessas populações seja dependente de fontes externas (OFFEl et al., 2014). Além disso, a produção de fertilizantes artificiais e a alteração dos ciclos biogeoquímicos constituem limites planetários ameaçados (STEFFEN et al., 2015).

Diante disso, deve-se pensar na implantação de sistemas de práticas adequadas, sobretudo nas zonas rurais, que possam contribuir não só com a proteção dos corpos hídricos, mas também com 0 aumento da produtividade agrícola em regiões semiáridas, onde há pouca disponibilidade de água e uma grande quantidade de pequenas propriedades, cuja produtividade ainda é baixa, principalmente por conta do alto custo de aquisição de adubos químicos. Nesse contexto, o saneamento ecológico é uma alternativa promissora na medida em que promove condições de higiene e a prevenção de doenças, bem como contribui para: a redução do uso e da importação de fertilizantes químicos, a mitigação da poluição ambiental, a preservação dos recursos hídricos, a fertilização do solo e o aumento na produção agrícola. Este tipo de tecnologia de saneamento representa uma metodologia de fluxo fechado que busca reintroduzir os nutrientes das águas residuais na agricultura, em vez de lançá-los nos corpos d'água, como é o padrão atual (SIMHA; GANESAPILLAI, 2017).

Uma premissa do saneamento ecológico é a segregação das correntes que constituem o esgoto segundo suas características e potenciais usos. No caso da urina humana, denominada águas amarelas, encontram-se $90,0 \%$ dos nutrientes excretados, podendo produzir cerca de $5,6 \mathrm{~kg}$ de nitrogênio, $0,4 \mathrm{~kg}$ de fósforo e 1,0Kg de potássio em um ano (LIND; BAN; BYDÉN, 2001). Tais características direcionam essa corrente para a sua reinserção no ciclo dos nutrientes, visando à produção de alimentos. Apesar das vantagens, a utilização de urina ainda enfrenta barreiras relacionadas a aspectos psicossociais e que precisam ser superadas, sendo, por isso, necessária uma melhor compreensão do problema.

Apesar de uma aceitação moderada, as pessoas acreditam que a urina humana tem 
risco à saúde e pode ser nociva às plantas, segundo Lienert et al. (2003); Matsebe e Moilwa (2007); Duncker e Matsebe (2008); Mariwah e Drangert (2011); Okem et al. (2013); Nimoh et al. (2014a, 2014b); Mugivhisa e Olowoyo (2015); Simha et al. (2018). Para os agricultores, pouco mais da metade pensa que esta é uma opção viável, (LIENERT et al., 2003; LONG, 2011; MÜLLER; PEDRO; FREITAS, 2017; SIMHA et al., 2018; TAHER et al., 2018), porém, o nível da aceitação do consumo de produtos das plantas fertilizadas com urina humana é, na maioria das vezes, inferior ao da aceitação de plantas fertilizadas por produtos convencionais (MAGIVHISA; OLOWOYO, 2015; IGNACIO et al., 2018; SIMHA et al., 2018).

Quanto à aceitação dos alimentos fertilizados com excreta humana (fezes e urina), as percepções dos agricultores são controversas, diversas e, às vezes, caracterizadas por alguns fenômenos culturais e sociais, como preocupações pessoais, preconceito, tabu, religião, risco à saúde etc. Também, as práticas de saneamento ambiental incluem vários fatores, tais como atributos sociais, econômicos e demográficos, como idade, renda, gênero, educação, estruturas domésticas, condições situacionais (DARAMOLA; OLOWOPOROKU, 2016; MUGIVHISA; OLOWOYO, 2015) e as atividades econômicas da população.

No Brasil, ainda são muito poucas as iniciativas para implantação de saneamento ecológico, bem como pesquisas de aceitação do uso de excretas como fertilizantes. Para um melhor entendimento das diferentes barreiras desta prática, é necessário conhecer melhor as percepções dos agricultores familiares, tanto em relação à aceitação da utilização de urina humana como fertilizante, quanto ao consumo das plantas fertilizadas dessa forma. Assim, este estudo teve como objetivo geral avaliar as percepções dos agricultores familiares da zona rural do semiárido baiano a respeito do uso de urina humana na agricultura.

\section{MATÉRIAS E MÉTODOS}

A pesquisa teve como objeto de estudo o uso de urina por parte dos agricultores familiares residentes no município de Serrinha, localizado no Estado da Bahia e inserido no Semiárido do Nordeste Brasileiro (Figura 1) entre as latitudes $11^{\circ} 39^{\prime} 51^{\prime \prime}$ Sul e longitude $39^{\circ} 00^{\prime} 27^{\prime \prime}$ Oeste. De acordo com o Censo de 2010 (IBGE, 2018), sua população em 2010 era de 76.762 habitantes e, para 2018, foi estimada em 80.411 habitantes. Possui área de $613,7 \mathrm{~km}^{2}$ e distância da capital (Salvador) de $180 \mathrm{~km}$ e de $70 \mathrm{~km}$ da cidade de Feira de Santana. A elevação em relação ao nível do mar é de $379 \mathrm{~m}$ de altitude. A agricultura familiar neste município apresenta uma das maiores fontes de renda e de sobrevivência. Sua principal atividade econômica é a agropecuária, destacando no setor agrícola as culturas do feijão, milho e mandioca e, na pecuária, a criação de bovinos e caprinos. O clima varia de semiárido ao subúmido e tropical, com variação de temperatura, no verão, de 24 a $32^{\circ} \mathrm{C}$ e, no inverno, de 14 a $28^{\circ} \mathrm{C}$, e pluviometria anual média de $801 \mathrm{~mm}$.

A escolha do município se deu em razão da acessibilidade aos agricultores familiares por meio da articulação com a Secretaria de Agricultura e, também, devido à relação de três dos entrevistadores participantes na pesquisa com os agricultores. Outro fator considerado foi o potencial do município para a agricultura familiar, o que possibilita a integração e a utilização da urina nas atividades agrícolas já desenvolvidas. A ação juntamente com a Secretaria de Agricultura no município permitiu o repasse de informações referentes aos agricultores familiares da região. O público entrevistado se limitou ao chefe de cada família agricultora.

\section{Coleta dos dados}

A coleta de dados foi feita por meio de entrevistas, com um questionário semiestruturado, realizadas com o(a)s responsáveis pela gestão extradomiciliar. 0 questionário continha vinte oito perguntas com respostas fechadas e com a opção de se preencher com uma ou outra resposta. Os assuntos abordados no questionário foram agrupados em 4 (quatro) conjuntos de 
perguntas. No primeiro conjunto (5 perguntas), continha temas relacionados a aspectos sociodemográficos, tais como sexo, faixa de idade, religião e nível de escolaridade dos entrevistados. No segundo (5 perguntas), foram informações referentes às atividades econômicas, com ênfase nos cultivos praticados, os tipos de animais criados, os locais de plantações e de criatórios de animais. O terceiro conjunto possuía 15 perguntas que buscavam identificar atitudes e percepções quanto ao uso de esterco e urina de animais e urina humana na agricultura, bem como verificar o conhecimento prévio do uso da urina humana como fertilizante, os motivos para não usar e aceitação segundo a origem da urina. No quarto e último conjunto, procurou-se perceber a diferença de respostas com relação ao uso de fertilizantes, químicos e orgânicos. Inicialmente, apresentaram-se ao entrevistado 6 (seis) frascos (sem identificação do seu teor), com diferentes conteúdos (Urina Humana Pura; Torta de Mamona; Urina Humana com Cinza; Urina Humana Diluída; Ureia e Cinza Pura) e, então, solicitado que o participante indicasse quais produtos (dos frascos) usaria como fertilizantes. Em seguida, foi feita a mesma pergunta, dessa vez, informando o conteúdo de cada frasco.

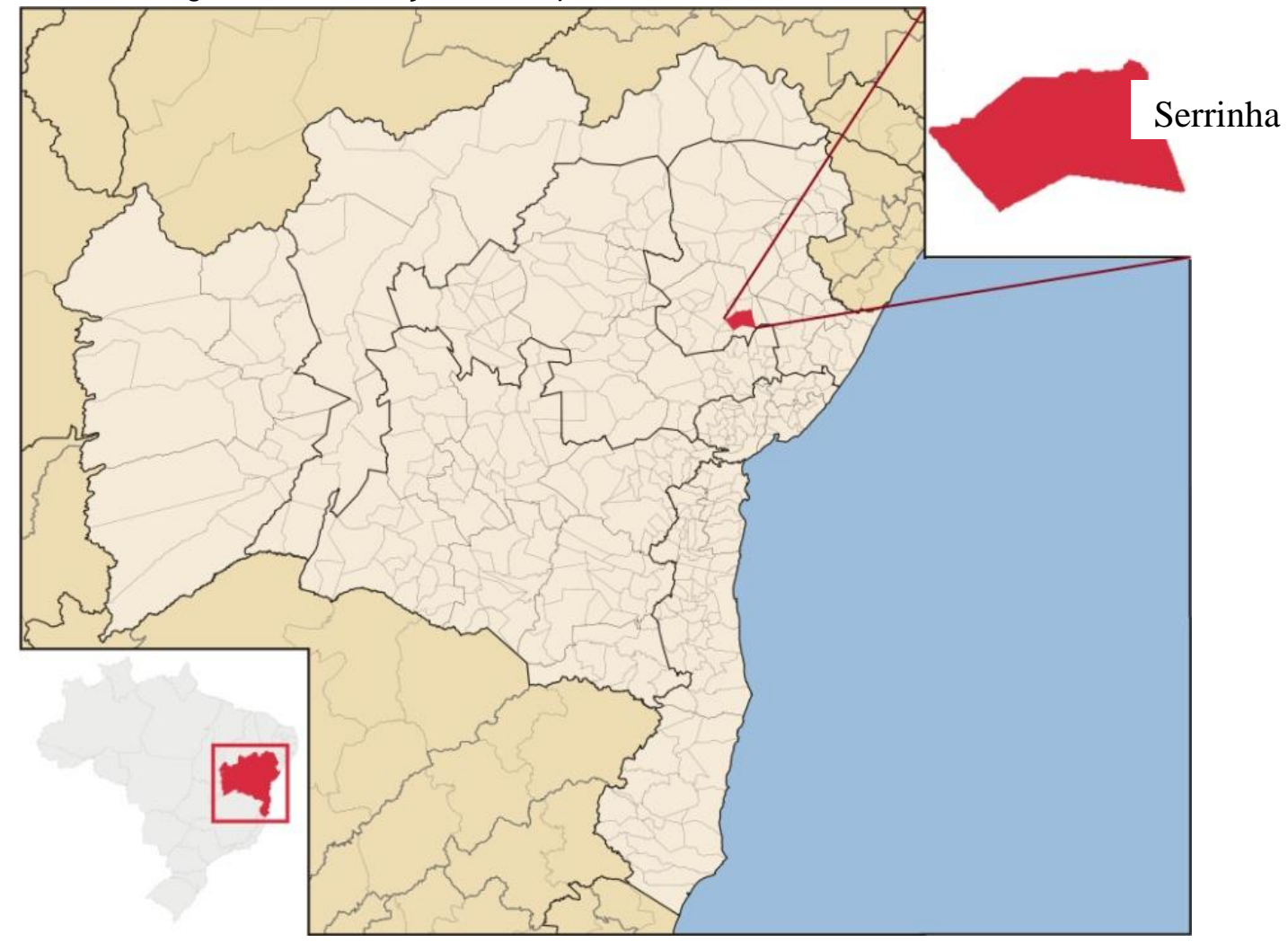

Fonte: adotada pelos autores, 2018.

Devido ao fato de que, geralmente, são os chefes da casa (mulheres ou homens) que decidem quais alimentos serão consumidos pela família e, muitas vezes, suas decisões são irrevogáveis, as entrevistas foram feitas com esses chefes. Para preservar sua identidade, a cada um foi atribuído um número. As respostas foram registradas em papel e revisadas depois da redação, a fim de garantir a estrita opinião de cada entrevistado.

A coleta de dados deu-se com aplicação de questionário em 210 agricultores familiares, em suas casas, escolhidos aleatoriamente, quando foi esclarecido o objetivo da entrevista e 
informada a importância da pesquisa no contexto de saneamento rural. O tempo médio de duração da aplicação do questionário foi de 12 minutos, depois de cada agricultor ter dado seu consentimento.

\section{Aspectos éticos}

Esta pesquisa foi aprovada pelo Comitê de Ética em Pesquisa - CEP da Universidade Estadual de Feira de Santana (UEFS), com o Registro 2.687.736. Antes da entrevista, apresentou-se a pesquisa informando seus objetivos e lido o Termo de Consentimento Livre e Esclarecido (TCLE), o qual, após o entendimento dos seus propósitos e da sua concordância, foi assinado pelo entrevistado ou por outra pessoa da família, no caso daquele ser analfabeto.

\section{Análises dos dados}

As análises dos dados foram realizadas em duas etapas: na primeira, analisaram-se as respostas das perguntas fechadas ('sim' e 'não') e, na segunda, as das questões abertas.

Para as respostas 'sim' e 'não', foi atribuído o número 1 e 0 , respectivamente, e para as perguntas com múltiplas escolhas, números que variam de 0 a 9 . Esse procedimento foi adotado para possibilitar a análise estatística.

A sistematização dos dados efetuou-se com o auxílio do programa $\mathrm{R}$ (versão 3.4.3). O teste de proporção foi usado para verificar se houve diferença significativa no nível de 0,05 , entre as variáveis sociodemográficas, para as questões apresentadas.

\section{RESULTADOS E DISCUSSÃO}

\section{Características socioeconômicas dos entrevistados}

A maioria dos entrevistados era do sexo feminino, com mais de 30 anos, de religião católica e estudou até 0 Fundamental Incompleto (Tabela 1).

O uso de fertilizantes é moderado, em geral, se limitando aos cultivos realizados nos quintais, e é adotado por $90,0 \%$ dos agricultores. Dentre os adubos mais utilizados, destaca-se o esterco de animais, usado puro $(46,2 \%)$ ou associado a outros fertilizantes, como os químicos, composto orgânico e resíduo de sisal $(8,2 \%)$.

Além dos cultivos de hortícolas, plantas frutíferas etc., para consumo próprio, as principais culturas desenvolvidas, conforme informaram os agricultores, foram o milho, feijão e mandioca (Tabela 2).

\section{Percepções dos agricultores sobre o uso de estercos e urina de animais}

Ao serem questionados se urina e estercos de animais são fertilizantes, os agricultores concordam que os estercos de bovinos, ovinos, caprinos e galináceos são fertilizantes, todos com percentuais de aceitação superiores a $90,0 \%$. Entretanto, o esterco de cavalo foi mencionado por $21,0 \%$, enquanto que os de cachorro, gato, porco e outros receberam, juntos, a menção de $18,0 \%$ (Figura 2). Não se identificou influência das variáveis sociodemográficas na crença de que estercos de animais são adubos.

Consultados quanto ao uso da urina dos mesmos animais como fertilizante, o nível de concordância se reduz significativamente. As maiores proporções, 55,2\%, 37,6\% e 35,7\%, respectivamente, referem-se à urina de bovino, caprino e ovino. As urinas de cavalo e de galinha foram referidas, respetivamente, por $6,2 \%$ e $11,4 \%$ dos entrevistados (Figura 2 ).

Referente à aceitação de estercos de galinha nesta pesquisa, o resultado é similar ao obtido por Mariwah e Drangert (2011) em pesquisa realizada em Gana, na qual verificaram que todos os entrevistados acreditam que estercos de animais podem ser usados como fertilizantes, principalmente os de galinha, que foram aceitos por $94,0 \%$ dos entrevistados, e $60,0 \%$ deles já os utilizam em suas plantas cultivadas.

A redução dessas proporções em relação às dos estercos pode estar associada à dificuldade de sua coleta e, sobretudo, devido ao fato de que a prática do uso de urina de animais não é tão comum quanto a do uso do esterco. Este resultado refere-se a uma 
percepção social. Segundo Khalid (2018) e Rosenquist (2005), a percepção humana se associa às culturas mais praticadas na sociedade.

No caso da urina de cavalo, $45,0 \%$ dos entrevistados acreditam que é perigosa para plantas, pois "colocando-a, a planta morrerá". Quanto à urina de galinha, esta foi vista como uma piada pelos entrevistados. Cerca de $90,0 \%$ reafirmaram a fala de: "a galinha não urina já que ninguém vê uma galinha que urina”. Para outros, a urina de galinha se mistura com suas fezes. Alguns agricultores referiram-se ao uso medicinal da urina humana, indicando aplicações para o tratamento alternativo de inchaço na barriga, para curar ferimentos e para conjuntivite. Segundo um entrevistado, um copo de urina misturado com alho e com cinza é bom para curar acidente vascular cerebral. Outros informaram que a urina humana é útil para matar pragas nas plantas, mencionado também por Long (2011) em estudo realizado na Belize. E outros relataram que "beber um copo de urina de vaca misturada com o leite dela é muito bom".

Tabela 1: Características sociodemográficas dos agricultores do município de Serrinha, 2018

\begin{tabular}{cccc}
\hline Característica & & Quantidade & Percentual (\%) \\
\hline \multirow{2}{*}{ Sexo } & Feminino & 126 & 60,0 \\
& Masculino & 84 & 40,0 \\
\hline \multirow{4}{*}{ Faixa de idade } & Até 30 anos & 51 & 24,3 \\
& 31 a 40 anos & 35 & 16,7 \\
& 41 a 60 anos & 73 & 34,7 \\
& 61 ou mais & 51 & 24,3 \\
\hline \multirow{3}{*}{ Religiões } & Católica & 168 & 80,0 \\
& Evangélica & 27 & 12,9 \\
& Sem & 14 & 6,6 \\
& Outras & 1 & 0,5 \\
\hline \multirow{3}{*}{ Escolaridade } & $\mathrm{Fl}^{*}$ & 141 & 67,2 \\
& $\mathrm{FC}^{*}$ até MI* & 45 & 21,4 \\
& $\mathrm{MC}^{*}$ ou mais & 24 & 11,4
\end{tabular}

$\mathrm{Fl}^{\star}$ : Fundamental Incompleto, ${ }^{*} \mathrm{FC}$ : Fundamental Completo, ${ }^{*} \mathrm{MI}$ : Ensino Médio Incompleto, ${ }^{*} \mathrm{MC}$ : Ensino Médio Completo

Fonte: elaborada pelos autores, 2018.

Tabela 2: Culturas cultivadas pelos agricultores

\begin{tabular}{ccc}
\hline Culturas & Quantidade & Percentual (\%) \\
\hline Milho & 205 & 97,6 \\
Feiião & 202 & 96,2 \\
Fruta & 179 & 85,2 \\
Hortaliça & 140 & 66,7 \\
Mandioca & 120 & 57,1 \\
Tomate & 105 & 50,0 \\
Melancia & 87 & 41,4 \\
\hline
\end{tabular}

Fonte: elaborada pelos autores, 2018. 
Figura 2: Estercos e urina de animais que os agricultores do município de Serrinha acreditam que são fertilizantes, 2018

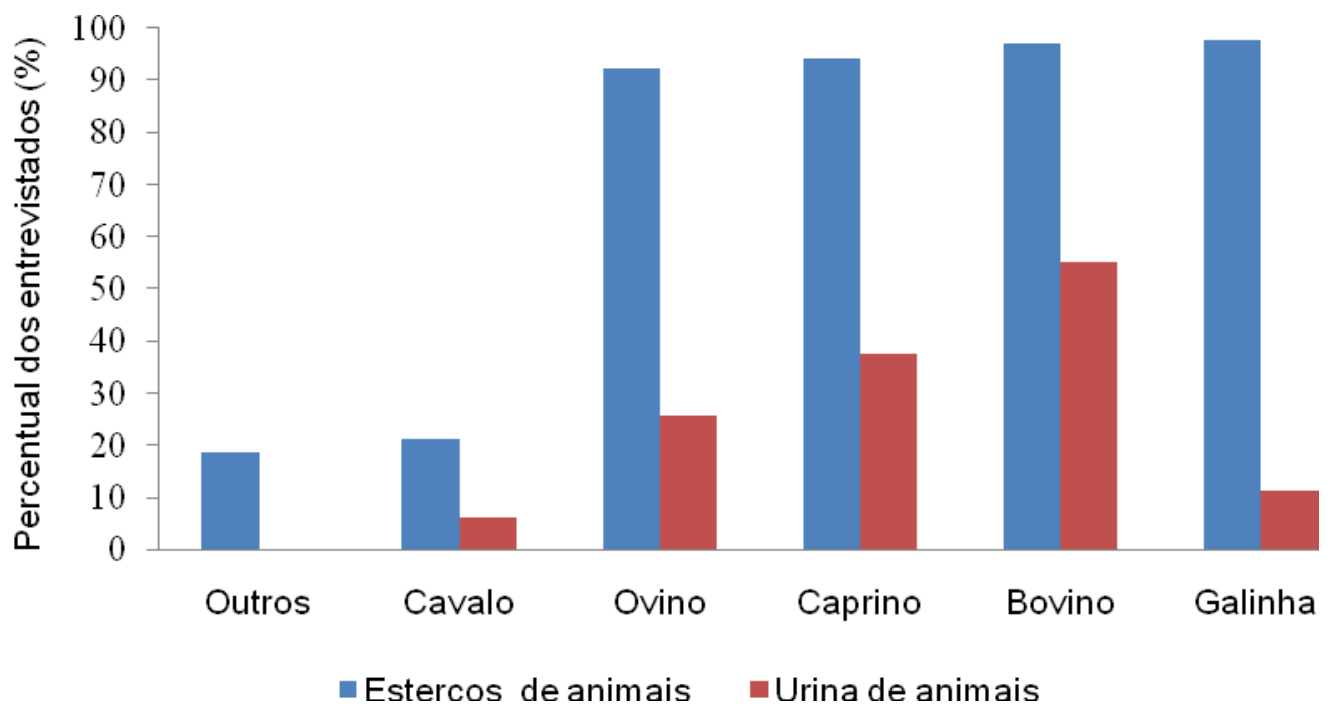

Fonte: elaborada pelos autores, 2018.

\section{O uso de urina humana na agricultura}

O uso agrícola da urina humana é uma prática pouco conhecida pelos agricultores entrevistados nesta pesquisa. Apenas 10,0\% deles já tinham ouvido falar do uso de urina como fertilizante. Resultados semelhantes foram apresentados por Müller, Pedro e Freitas (2017) em Tefé, na Amazônia Central, com 12,0\%. Ressalta-se a diferença cultural entre essas regiões do país. Em algumas sociedades, o uso de excreta humana na agricultura é milenar, a exemplo da China, onde os agricultores chineses a consideram como um bem precioso (KHALID, 2018).

Apesar de pouco conhecido o uso de urina como fertilizante, $67,7 \% \quad(67,9 \%$ homens e $67,5 \%$ mulheres) dos agricultores entrevistados afirmaram que a usaria se tiverem conhecimento de como proceder, enquanto $32,3 \%$ agricultores relataram que não usaria porque outras pessoas não usam. Encontrou-se na literatura relatos de aceitação do uso de excreta humana com percentuais variados. Conforme estudos realizados em diferentes lugares por Khalid (2018), Rosenquist (2005), Peng, Chang e Zhou (2013), os obstáculos a isso são influenciados por aspectos psicológicos, demográficos e socioculturais, com comportamentos distintos de uma sociedade para outra.

O percentual de aceitação do uso de urina pelos entrevistados nesta pesquisa é superior ao encontrado no estudo feito em Tefé, na Amazônia Central, onde 47,0\% dos entrevistados afirmaram que usaria urina humana na agricultura (MÜLLER; PEDRO; FREITAS, 2017). Em pesquisas semelhantes, Simha et al. (2018), no sul da Índia, e Taher et al. (2018), no Iraque, Egito e Turquia, encontraram, respectivamente, uma aceitação por parte de $55,0 \%$ e $56,0 \%$ dos entrevistados.

Um aspecto relevante, quanto à aceitação da urina humana para o uso agrícola entre estes agricultores familiares, refere-se à proximidade $e$ ao agente produtor da urina, conforme mostrado na Figura 3, em que se encontraram pessoas que usaria tanto suas próprias urinas, incluindo as de membros de suas famílias, quanto urinas de pessoas desconhecidas. Um percentual de $66,7 \%$ dos agricultores usaria apenas suas próprias urinas, enquanto $41,4 \%$, suas urinas e as de seus familiares, e apenas $8,6 \%$ fariam uso de urina de pessoas desconhecidas (Figura 3) $(p$-valor $<0,05)$. Assim, à medida que a origem da 
urina se afasta de seus familiares, sua aceitação se torna mais difícil. O mesmo comportamento foi verificado em uma pesquisa na África do Sul, onde $53,3 \%$ dos entrevistados declararam que usaria a urina de suas famílias contra $20,5 \%$ que usaria de outras pessoas (OKEM et al., 2013).

Um dos motivos alegados para não usar urina de outras pessoas foi o risco de doença "devido às bactérias que podem existir na urina, principalmente quando a bexiga de uma pessoa não descarrega urina após relações sexuais" (Entrevistado 42). No presente estudo, alguns entrevistados comentaram que a urina humana é nociva para as plantas, da mesma forma que é mencionado por outros autores em estudos semelhantes, a exemplo de Matsebe e Moilwa (2007) e Duncker e Matsebe (2008).

Observa-se que não há variações expressivas entre os níveis de aceitação da urina segundo a idade de quem a produz, embora haja uma pequena desvantagem para as urinas de jovens $(45,7 \%)$ e de idosos $(45,7 \%)$ quando comparadas às de crianças $(51,0 \%)$ e de adultos (52,4\%) (Figura 4).
Neste estudo, alguns dos entrevistados afirmaram que não usaria a urina de jovens, porque eles "mexem em muitas coisas" que podem afetar negativamente a qualidade de suas urinas, numa alusão a maior promiscuidade das práticas sexuais. Quanto à urina dos idosos, alguns apontaram que o cheiro é muito forte.

A utilização de urina como adubo nas plantas frutíferas arbóreas, cujo fruto não tem contato com o solo, foram mais aceitas $(39,5 \%)$ do que aquelas com frutos em contato direto com o solo, como abóbora $(28,1 \%)$ e melancia $(26,7 \%)$ ( $p$-valor varia de 0,005 a 0,013) (Figura 5). Do mesmo modo, os cultivos cujos produtos são consumidos cozidos, caso do feijão $(38,1 \%)$, milho $(39,1 \%)$ e mandioca $(36,2 \%)$, seriam mais facilmente adubados com urina que aqueles consumidos crus. A mesma observação foi feita num estudo semelhante realizado no Paquistão por Khalid (2018), no qual se constatou que a comunidade estava pronta para reutilizar a excreta para culturas anuais (milho, trigo, cevada etc.), mas não para horticultura.

Figura 3: Aceitação do uso de urina humana na agricultura segundo sua origem

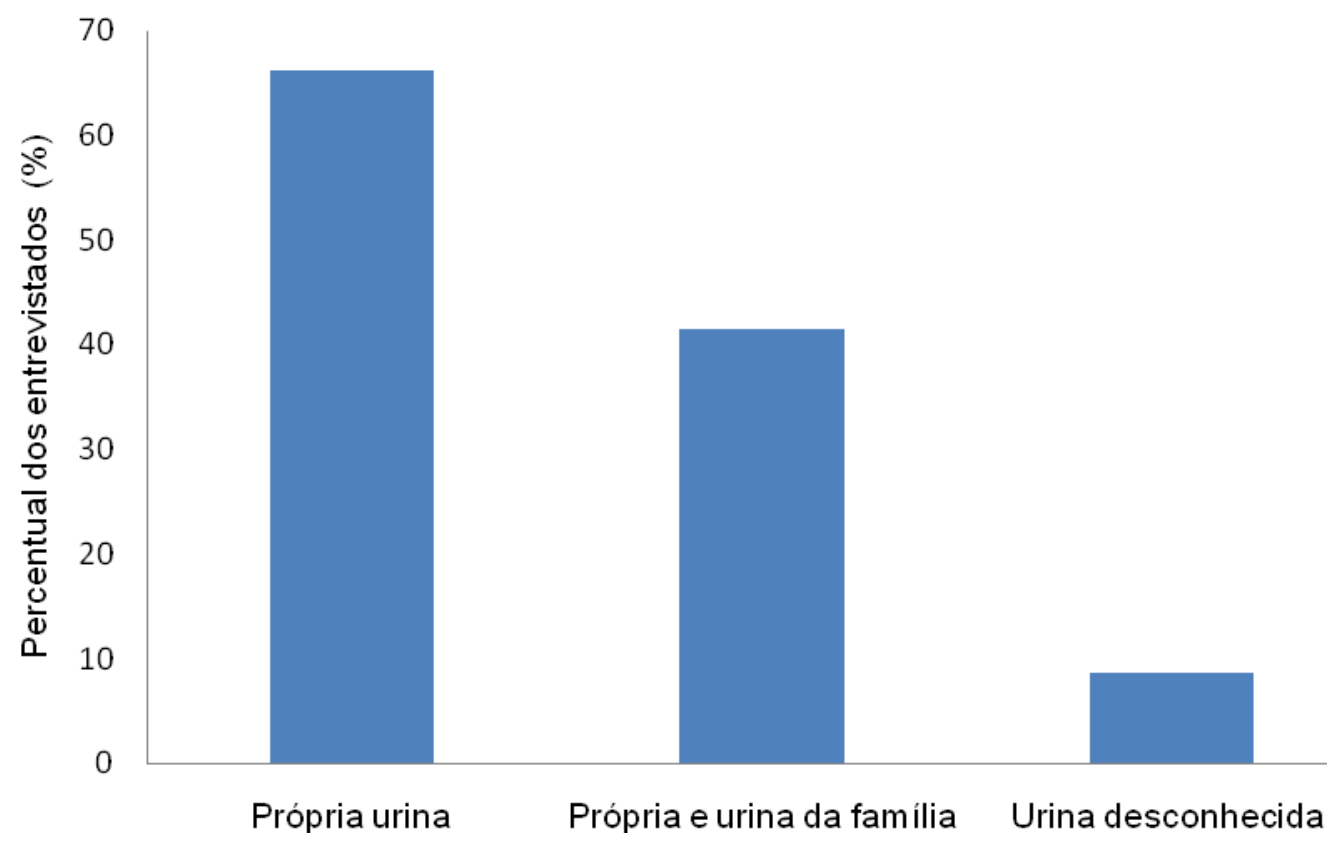

Fonte: elaborado pelos autores, 2018. 
Figura 4: Aceitação do uso de urina humana segundo a faixa etária do fornecedor

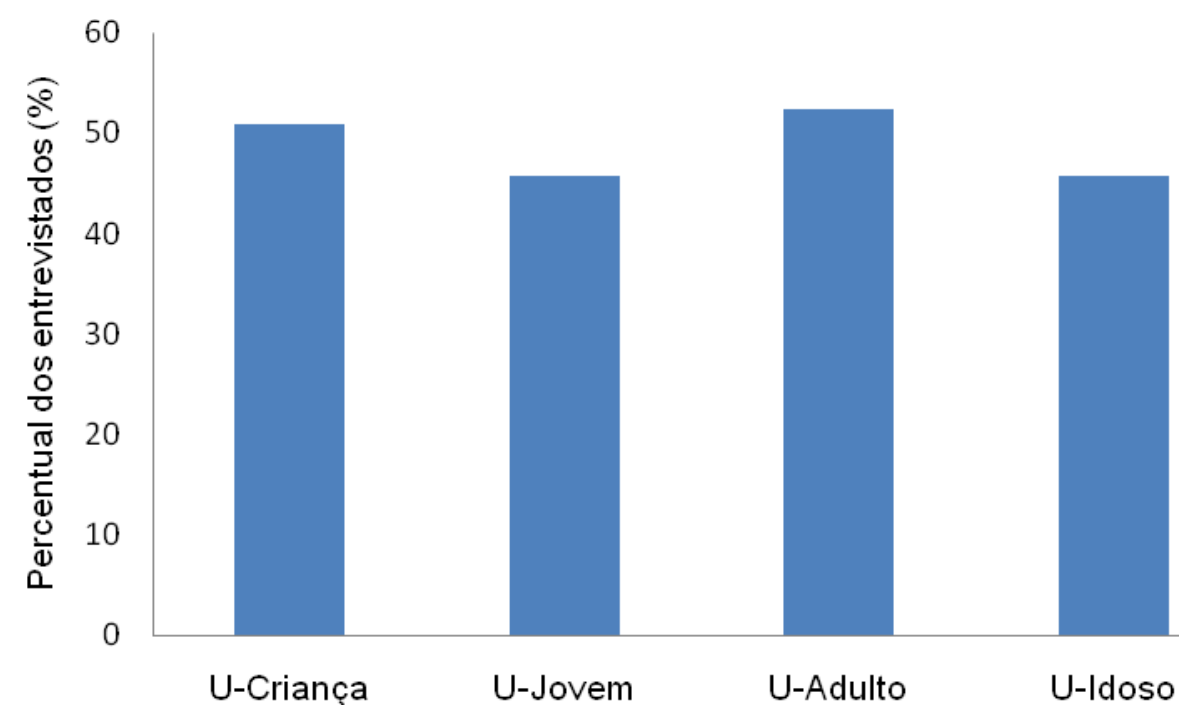

Fonte: elaborado pelos autores, 2018.

Figura 5: Aceitação dos tipos de plantas fertilizadas com urina humana e o seu consumo

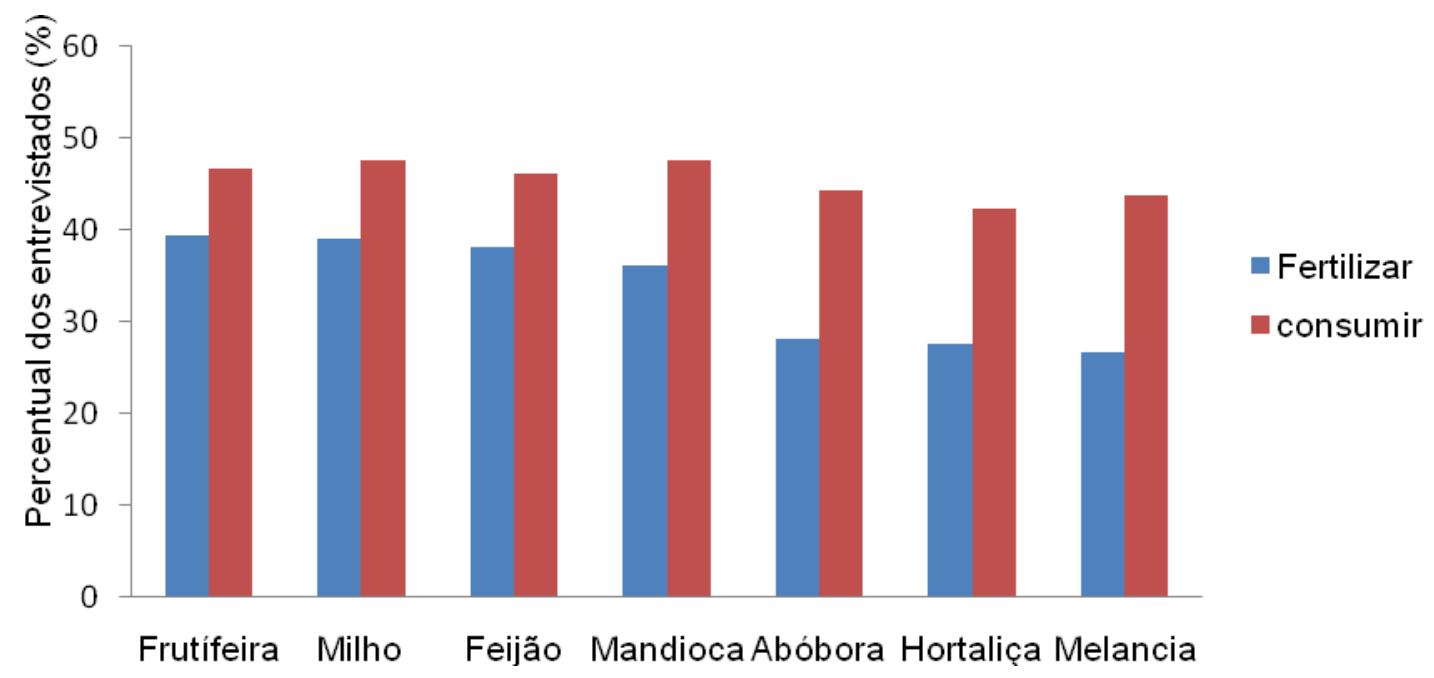

Fonte: elaborada pelos autores, 2018.

Quando questionados sobre a disposição de consumir os produtos fertilizados com urina, observa-se uma aceitação média maior para todos os cultivos $(45,5 \%)$ que a de adubar
(33,6\%). Nota-se também que, nesse aspecto, o comportamento foi mais uniforme, com um coeficiente de variação de 4,0\% para consumir, contra $17,0 \%$ para adubar. Esse resultado, até 
certo ponto surpreendente, talvez possa ser explicado pelo fato de que fertilizar requer o manejo da urina enquanto que consumir, não (Figura 5).

Não foram identificadas diferenças significativas entre as categorias sociodemográficas analisadas para os diversos cultivos a serem fertilizados com urina humana, exceto para hortaliça, cuja aceitação foi maior entre católicos $(31,6 \%)$ do que entre os evangélicos $(11,1 \%)$ ( $p$ com valor de 0,029) (Tabela 3 ).
Do mesmo modo, verificou-se uma maior aceitação entre os agricultores com escolaridade equivalente a Médio Completo ou Superior $(45,8 \%)$ do que entre os com escolaridade menor ou igual a Fundamental Incompleto (33,3\%), com p-valor=0,016 (Tabela $3)$.

As principais restrições ao uso da urina como fertilizante apontadas pelos entrevistados foram o risco à saúde, mau cheiro e nojo (Figura $6)$.

Tabela 3: Aceitação de fertilizar a hortaliça com urina humana

\begin{tabular}{lccc}
\hline $\begin{array}{l}\text { Característica } \\
\text { Sóciodemograticas }\end{array}$ & & Percentual (\%) & p-valor \\
\hline \multirow{2}{*}{ Religiões } & Católica & 31,6 & \multirow{2}{*}{0,029} \\
\hline Escolaridade & Evangélica & 11,1 & \\
& Até Fl* & 33,3 & \multirow{2}{*}{0,016} \\
& MC* ou mais $^{*}$ & 45,8 & \multirow{2}{*}{ o, }
\end{tabular}

Fonte: elaborada pelos autores, 2018.

Figura 6: Motivos para não usar os produtos fertilizados com urina humana

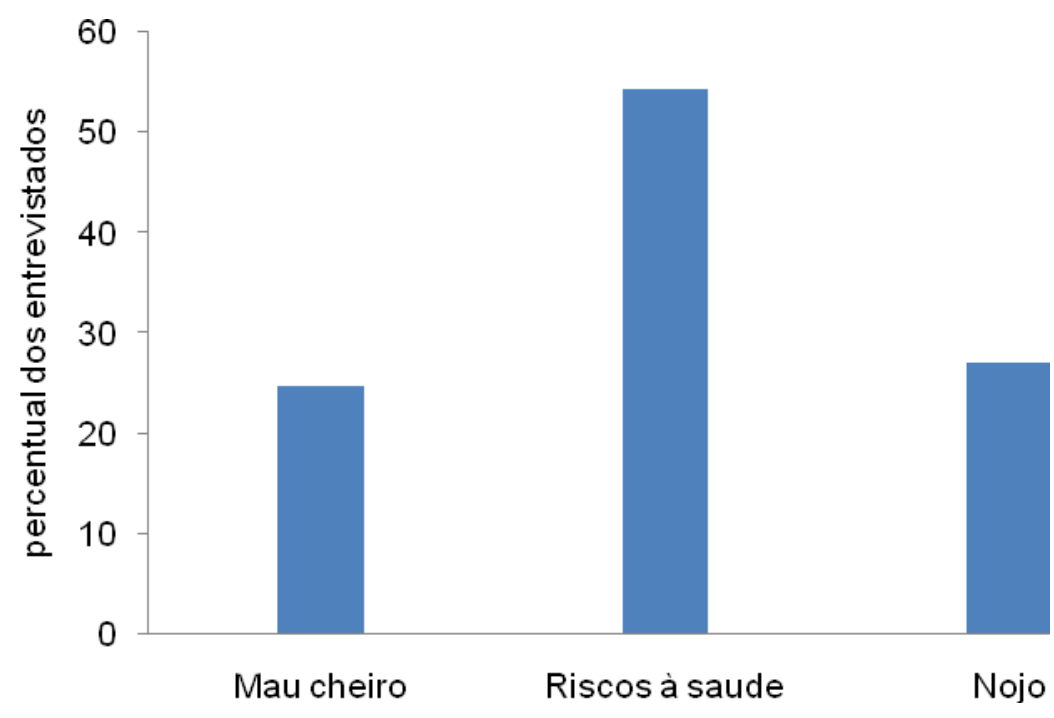

Fonte: elaborada pelos autores, 2018.

A maioria $(54,3 \%)$ não usaria a urina como fertilizante por acreditarem que existe risco à saúde (Figura 6). Estudos realizados na África do Sul por Mugivhisa e Olowoyo (2015), em Gana por Nimoh et al. (2014a, 2014 b) e na Índia por Simha et al. (2018) mostram que cerca 
de $65,0 \%$ dos entrevistados apontam o risco à saúde como a principal restrição ao uso da urina. Houve maior proporção entre as mulheres do que entre os homens.

$\mathrm{Na}$ categoria idade, não houve diferenças significativas para os fatores mau cheiro e risco à saúde, mas para o fator nojo, sim, entre as faixas etárias de 41-60 (31,5\%) e mais de 61 anos $(9,8 \%)$ ( $p$-valor $=0,004)$. De acordo com o nível de estudo, houve diferença significativa para o fator risco à saúde entre $\mathrm{FI}(55,3 \%)$ e $\mathrm{MC}$ ou mais $(33,3 \%)$ ( $p$-valor $=0,046)$ e entre $\mathrm{FC}-\mathrm{Ml}$ $(62,2 \%)$ e $M C$ ou mais $(33,3 \%)$ ( $p$-valor $=0,022)$. Neste estudo, a maioria dos entrevistados que se preocupou com o risco à saúde decorrente do uso da urina humana manifestou também nojo e preocupação em relação ao mau cheiro da urina.

Segundo Peng, Chan e Zhou (2013), o nojo de excreta humana é, na maioria das vezes, relacionado ao risco à saúde, visto que se sente nojo de tudo que possa representar ameaça para sua saúde, como é caso da urina, sobretudo a de outras pessoas.

Quando da apresentação dos 6 (seis) frascos com diferentes conteúdos (mistura de cinza com urina humana, urina humana pura; urina humana diluída, torta de mamona; cinza pura e ureia) sem identificação, Ihes foi perguntado quem deles utilizaria 0 mesmo frasco depois de terem a informação sobre a natureza do seu conteúdo. Houve uma aceitação crescente dos fertilizantes apresentados às cegas, na seguinte ordem: urina pura e urina diluída $(19,1 \%)$, urina com cinza $(40,5 \%)$, cinza pura $(46,7 \%)$, ureia $(47,1 \%)$ e torta de mamona $(76,2 \%)$ (Tabela 7$)$. Não houve diferença significativa entre urina pura e a diluída, bem como entre urina com cinza, cinza pura e ureia. Para as diversas categorias sociodemográficas, não se verificaram diferenças significativas.

Com o conteúdo revelado, a ordem de preferência se manteve, embora tenha se observado um crescimento na disposição para usar todos os produtos apresentados, conforme mostrado na Figura 7, na qual se observam os maiores crescimentos no percentual de aceitação para a urina pura, urina diluída e cinza, respectivamente, $62,0 \%, 70,0 \%$ e $64,0 \%$. Para as diversas categorias sociodemográficas, também não se verificaram diferenças significativas.

Esse crescimento pode ser explicado pelo medo do desconhecido, caso em que se enquadra a escolha às cegas. É importante destacar que quando perguntados sobre se usariam a urina como fertilizante, $67,6 \%$ responderam afirmativamente, contudo, quando apresentados à amostra de urina pura ou diluída, esse percentual ficou em torno de $31,5 \%$, menos da metade da manifestação de intenção de uso.

Outro aspecto a ser destacado é que a urina misturada com cinza teve uma aceitação $74,0 \%$ maior do que a urina pura ou diluída. Isso aponta para o fato de que a descaracterização da urina enquanto excreta pode ser uma importante estratégia para a popularização de seu uso como fertilizante.

O elevado nível de aceitação da ureia, que chegou a $61,9 \%$ no teste revelado, sugere que outra estratégia na recuperação dos nutrientes da urina pode ser a produção de estruvita, cujo seu aspecto é semelhante.

\section{CONCLUSÕES E RECOMENDAÇÕES}

Apenas $10,0 \%$ dos agricultores familiares do município da pesquisa tinham já ouvido falar do uso de urina humana na agricultura. Cerca de dois terços $(67,6 \%)$ de todos entrevistados responderam positivamente quanto ao uso da urina como fertilizante, porém, quando Ihes foram apresentados a urina concretamente, este percentual caiu para menos da metade $(31,5 \%)$. Observou-se também que a urina com cinza apresentou um crescimento de $74,0 \%$ maior do que a urina pura.

Os fatores sociodemográficos influenciaram pouco nas decisões dos agricultores na aceitação do uso de urina humana na agricultura. Suas principais preocupações foram risco à saúde $(54,3 \%)$, nojo $(27,1 \%)$ e mau cheiro $(24,8 \%)$. As culturas que não têm contato direito com a urina apresentaram maior aceitação tanto para fertilizar com a urina humana, quanto para consumir seus produtos 
crus ou cozidos. Assim, o uso de urina humana na agricultura foi aceito por estes agricultores, tornando uma alternativa viável que pode ser ampliada naquele município.

Recomenda-se desenvolver uma pesquisa experimental visando fertilizar algumas plantas com urina humana em zonas rurais, com a participação ativa dos agricultores, para que eles possam perceber que a urina é um excelente fertilizante compatível àqueles comumente utilizados por eles: os estercos de galinha e ruminantes.
Sugere-se divulgar informações por meio de televisão, rádio e pesquisas científicas sobre - aproveitamento de urina humana na agricultura, a fim de favorecer um melhor conhecimento desta prática e também minorar o paradigma comum dos agricultores de que a urina humana contamina as plantas, causando riscos à saúde. Aconselha-se apresentar aos agricultores um subproduto derivado de cinza com urina humana para usar na agricultura.

\section{Figura 7: Escolha dos frascos de fertilizantes sem e com conhecimento do conteúdo}

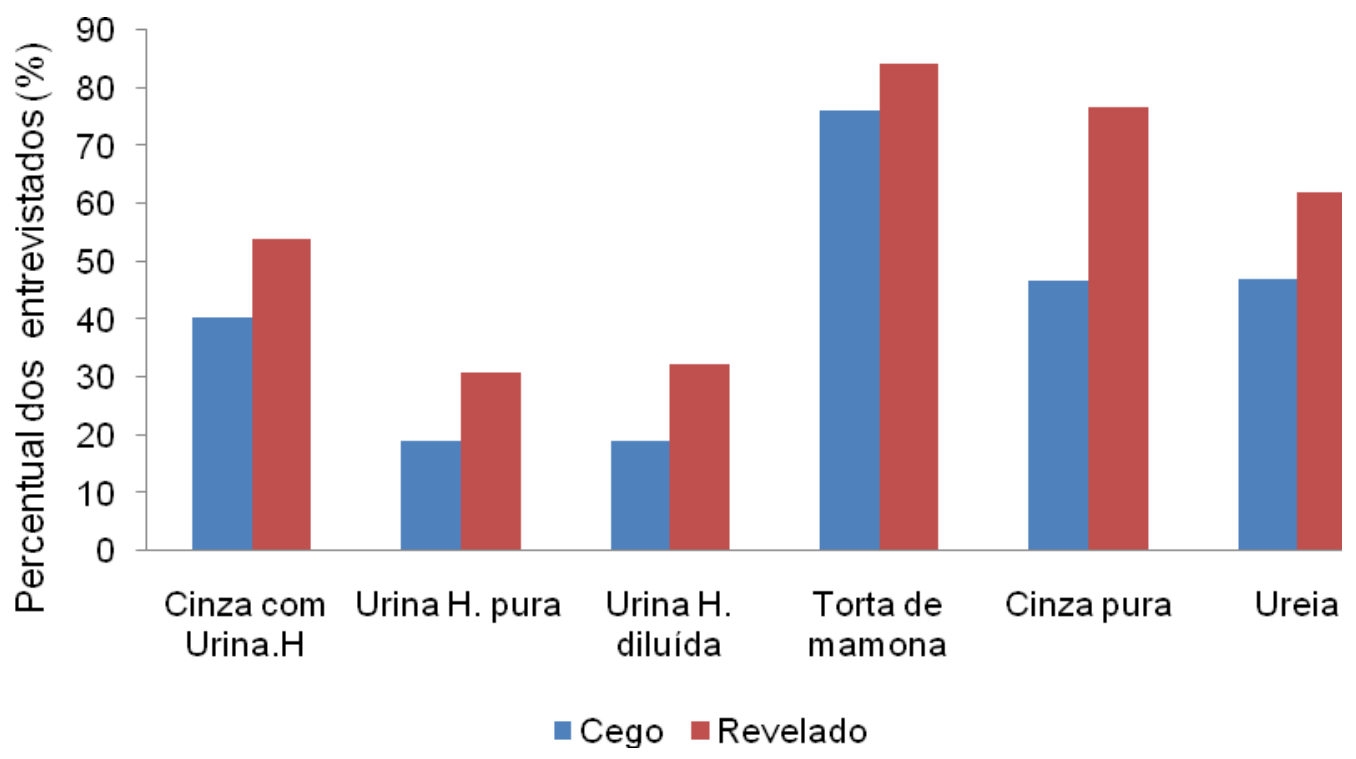

Fonte: elaborada pelos autores, 2018.

\section{REFERÊNCIAS}

DARAMOLA, O.; OLOWOPOROKU, O. Environmental sanitation practices in Osogbo, Nigeria: An assessment of residents' sprucing-up of their living environment. Osogbo: Economic and Environmental Studies, 2016. 18p.

DUNCKER, L. C.; MATSEBE, G. N. Prejudices and attitudes toward reuse of nutrients from urine diversion toilets in South Africa. Accra: 33 rd WEDC International Conference, 2008. 6p.

IBGE.Instituto Brasileiro de Geografia e Estatística.

População. Disponível em:
$<$ https://cidades.ibge.gov.br/brasi//ba/serrinha/panorama $>$ . Acesso em: dez. 2019.

IGNACIO, J.J.; MALENAB, R.A.; PAUSTA, C.M.; BELTRAN, A.; BELO, L.; TANHUECO, R.M.A.; ERA, M.; EUSEBIO, R.C.; PROMENTILLA, M.A.; ORBECIDO, A. Perceptions and attitudes toward eco-toilet systems in rural areas: A case study in the Philippines. Sustainability, v. 10, n. 2, p. 521, fev. 2018.

KHALID, A. Human excreta: a resource or a taboo? Assessing the socio-cultural barriers, acceptability, and reuse of human excreta as a resource in Kakul Village District Abbottabad, Northwestern Pakistan. Journal of 
Water Sanitation and Hygiene for Development, v. 8, n. 1, p. 71-80, mar. 2018.

LIENERT, J.; HALLER, M.; BERNER, A.; STAUFFACHER, M.; LARSEN, T.A. How farmers in Switzerland perceive fertilizers from recycled anthropogenic nutrients (urine). Water Science and Technology, v. 48, n. 1, p. 47-56, 2003.

LIND, B.-B.; BAN, Z.; BYDÉN, S. Volume reduction and concentration of nutrients in human urine. Ecological Engineering, v. 16, n. 4, p. 561-566, fev. 2001.

LONG, A. The uses of human urine and faeces in agriculture: Guidance for future experiments. Highland Heights: Nothern Kentuky University, 2011. 23p.

MARIWAH, S.; DRANGERT, J.-O. Community perceptions of human excreta as fertilizer in peri-urban agriculture in Ghana. Waste Management \& Research, v. 29, n. 8, p. 815-822, ago. 2011.

MATSEBE, G.N.; MOILWA, N. The social/cultural acceptability of using human excreta (faeces and urine) for food production in rural settlements in South Africa: Pretoria, report to the Water Commission, 2007.

MUGIVHISA, L.L; OLOWOYO, J.O. An assessment of university students and staff perceptions regarding the use of human urine as a valuable soil nutrient in South Africa. African Health Sciences, v. 15, n. 3, p. 999-1010, 2015.

MÜLLER, P.; BORGES PEDRO, J. P.; DE CASTRO FREITAS, C. H. Farmers' perceptions on the agricultural use of human urine in the Central Amazon. Mundo Amazónico, v. 8, n. 1, jan. 2017.

NIMOH, F.; OHENE-YANKYERA, K.; POKU, K.; KONRADSEN, F.; ABAIDOO, R.C. Farmers perception on excreta reuse for peri-urban agriculture in southern Ghana. Journal of Development and Agricultural Economics, v. 6, n. 10, p. 421-428, 1 out. 2014b.

Health

risk perception on excreta reuse for peri-urban agriculture in Southern Ghana. Shai-Osudoku: Journal of Economics and Sustainable Development, 2014a. 9p.

OFFEI, M.K.; EGYIR, I.S.; KWADZO,G.T-M.; COFIE,O. Financial feasibility of producing a urine-based fertilizer for vegetable farming in Accra, Ghana. Journal of Advances in Agricultural Science and Technology, v. 2, n. 1, p. 1-9, 2014.
OKEM, A.E.; XULU, S.; TILLEY, E.; BUCKLEY, C.; ROMA, E. Assessing perceptions and willingness to use urine in agriculture: A case study from rural areas of eThekwini Municipality, South Africa. Journal of Water, Sanitation and Hygiene for Development, v. 3, n. 4, p. 7, 2013.

PENG, M.; CHANG, L.; ZHOU, R. Physiological and behavioral responses to strangers compared to friends as a source of disgust. Evolution and Human Behavior, v. 34, n. 2, p. 94-98, mar. 2013.

ROSENQUIST, D.L.E. A psychosocial analysis of the human-sanitation nexus Louise Emilia. Journal of Environmental Psychology, v. 25, p. 335-346, 2005.

SIMHA, P.; GANESAPILLAI, M. Ecological sanitation and nutrient recovery from human urine: How far have we come? A review. Sustainable Environment Research, n. 27, p. 107-116, 2017.

LALANDER, C.; RAMANATHAN, A.; VIJAYALAKSHMI, C.; MCCONVILLE, J.R.; VINNERÅS, B.; GANESAPILLAI, M. What do consumers think about recycling human urine as fertilizer? Perceptions and attitudes of university community in South India. Water Research, n. 143, p. 527-538, 2018.

STEFFEN, W. ; RICHARDSON, K. ; ROCKSTRÖM, J. ; CORNELL,S.E.; FETZER, I.; BENNETT, E.M.; BIGGS, R.; CARPENTER, S.R.; DE VRIES, W.; DE WIT, C.A.; FOLKE, C.; GERTEN, D.; HEINKE, J.; MACE, G.M.; PERSSON, L.M.; RAMANATHAN, V.; REYERS, B.; SÖRLIN, S. Planetary boundaries: Guiding human development on a changing planet. Science, v. 347, n. 6223, p. 1259855, fev. 2015.

TAHER, M.N.; BASAR, A.; ABDELRAHMAN, A.M.; BELER-BAYKAL, B. Yellow water to aid food security: Perceptions/acceptance of consumers toward urine based fertilizer. Proceedings, v. 2, n. 11, p. 606, jul., 2018.

WEIDEMA, B. P.; FANTKE, P. Relating the global burden of disease to life cycles. Procedia CIRP, v. 69, p. 417422, 2018.

WORLD BANK. Atlas of sustainable development goals 2018: from world development indicators. 2018. 91p. 